\title{
PARAMETERS OF CURRENT SYSTEMS IN THE MAGNETOSPHERE AS DERIVED FROM OBSERVATIONS OF COSMIC RAYS DURING THE 2015 JUNE MAGNETIC STORM
}

\author{
G.N. Kichigin \\ Institute of Solar-Terrestrial Physics SB RAS, \\ Irkutsk, Russia, king@iszf.irk.ru
}

M.V. Kravtsova

Institute of Solar-Terrestrial Physics SB RAS, Irkutsk,Russia,rina@iszf.irk.ru

\author{
V.E. Sdobnov \\ Institute of Solar-Terrestrial Physics SB RAS, \\ Irkutsk,Russia,sdobnov@iszf.irk.ru
}

\begin{abstract}
Basing on measurements of cosmic rays at the worldwide network of stations, we calculate variations in the planetary system of geomagnetic cutoff rigidity for the 2015 June moderate geomagnetic storm. Using the axisymmetric model of the limited magnetosphere taking into account magnetopause currents and the ring current, we determine the distance to the subso-
\end{abstract}

lar point and the ring current radius, as well as the contribution of the ring current to variations in the geomagnetic cutoff rigidity and to the Dst index.

Keywords: magnetosphere, current system, magnetic storm, cosmic rays.

\section{INTRODUCTION}

The dynamics of large-scale current systems in the disturbed magnetosphere is still one of the open questions in solar-terrestrial physics.

A magnetic storm is a response of the magnetosphere to a sudden increase in the dynamic pressure of the solar wind (SW). It occurs with an intense energy release in the magnetosphere and ionosphere, which depends largely on the magnitude and direction of the interplanetary magnetic field (IMF).

During magnetic storms, the magnetic field depression at low latitudes increases sharply, being characterized by an increase in negative Dst. According to current views, its variations are strongly affected by the symmetrical ring current in the inner magnetosphere (DR), the transverse current in the magnetotail (DT), and the current at the magnetopause (DCF); hence $D s t=\mathrm{DR}+\mathrm{DT}+\mathrm{DCF}$ [Feldstein et al., 2005].

Magnetopause currents respond to changes in SW parameters. Compression of the magnetosphere when SW dynamic pressure rises leads to a short-term positive variation of the magnetospheric field in the inner and dayside magnetosphere (sudden storm commencement, SSC). The distance to the subsolar point at the magnetopause is one of the important magnetospheric parameters, which describes the magnetopause compression. Its variations at sudden jumps of SW dynamic pressure cause significant variations in the magnetospheric magnetic field.

Without regard to field-aligned currents, the current system of the magnetotail comprises currents across the magnetotail and triggering currents at the magnetopause.

The ring current is the most inert part of the magnetosphere. It is generated by injection of charged particles from the magnetotail and breaks down due to charge exchange with exospheric atoms, Coulomb collisions, and wave processes. Increased injection occurs during magnetospheric substorms, accompanied by strong dipolization of the tail's magnetic field. The magnetic field of the ring current is southward in the inner magnetosphere; it supports a characteristic large-scale structure of the variation of the measured magnetic field during a magnetic storm.

The relationship between Dst sources varies in different storm phases and can characterize the relative dynamics of magnetospheric current systems during disturbances [Alexeev et al., 1996; Maltsev et al., 1996; Greenspan, Hamilton, 2000].

Contributions to the Dst index from the DR and DT currents depend on storm intensity. For weak and moderate storms, the DT-current contribution may be as great as the DR-current contribution and sometimes may even exceed it. During strong storms, the ring current plays a more significant role. During moderate storms of about $-(100 \div 200) \mathrm{nT}$, the field of tail currents is saturated, reaching the maximum possible value, while the ring current is capable of developing further [Ganushkina et al., 2004; Kalegaev et al., 2005].

Dynamic processes occurring in the interplanetary medium and Earth's magnetosphere, due to their electromagnetic nature, affect charged cosmic-ray particles, producing variations in the spatial, angular, and energy distribution of the particles. Therefore, data on cosmic-ray $(\mathrm{CR})$ intensity variations can provide valuable information on electromagnetic conditions in outer space.

The structure of the total magnetic field in which CR particles move determines the CR planetary distribution and its time dependence on the geomagnetic field. Geomagnetic variations cause changes in asymptotic directions of arrival of particles at a point of observation as well as variations in the geomagnetic cutoff rigidity at this point, thus producing magnetospheric CR intensity variations, which are generally observed along with interplanetary $\mathrm{CR}$ variations during geomagnetic disturbances. 
To separate the contributions of interplanetary and magnetospheric CR intensity variations observed on Earth's surface, ISTP SB RAS has developed a spectrographic global survey (SGS) method adopted to gain information on primary CR distribution over energies and pitch angles in IMF and on variations in GCR planetary system per observation hour from ground-based CR observations at the worldwide network of stations [Dvornikov et al., 1984, 1986] and to analyze the entire available ground-based dataacquisition equipment (the worldwide network of neutron monitors located at any levels in Earth's atmosphere, ground and underground meson telescopes, etc.). This allows us, along with phases of the first and second harmonics of pitch-angle anisotropy, to determine the rigidity spectrum of the isotropic component and anisotropy from the pitch-angle anisotropy phase, to gain information on IMF orientation, as well as to determine variations of the GCR planetary system for each hour of observation or for shorter intervals.

Information on variations in the GCR planetary system during strong geomagnetic disturbances was used to test models of Earth's magnetosphere (see, e.g., [Tyasto et al., 2011, 2013]).

We examine variations in the GCR planetary system during the moderate geomagnetic storm in June 2015, using data on CR intensity from the worldwide network of stations. We employ an axisymmetric model of Earth's limited magnetosphere [Kichigin, Sdobnov, 2017], which takes into account magnetopause currents and ring current, to calculate the distance to the subsolar point and the ring current radius.

In the latter half of June 2015, in the active region (AR) 12371, one of the largest in the current solar cycle, there was increased solar activity [https:/www. solarmonitor.org]. In particular, on June 21 and 22, 2015, the AR gave two flares followed by full-halo CMEs about $360^{\circ}$ wide: the M2.0 flare $\left(12^{\circ} \mathrm{N}, 13^{\circ} \mathrm{E}\right)$ started at 02:06 UT on June 21,2015 , followed by CME with a velocity of $\sim 1400 \mathrm{~km} / \mathrm{s}$ at $02: 36 \mathrm{UT}$; the M6.5 flare $\left(18^{\circ} \mathrm{N}, 06^{\circ} \mathrm{W}\right)$ began at 17:39 UT on June 22, 2015, followed by CME with a velocity of about $1200 \mathrm{~km} / \mathrm{s}$ at 18:23 UT.

The interaction between magnetic clouds on June 21 and 22, when the subsequent cloud propagated along an already disturbed medium, caused a strong disturbance of the interplanetary medium in the vicinity of Earth and, as a consequence, the geomagnetic storm with maximum geomagnetic field depression on June 23, 2015 (the Dst index was -204 nT).

The worldwide network of neutron monitors on June 23, 2015 recorded a Forbush effect with $\sim 6-9 \%$ CR neutron component modulation amplitude.

\section{DATA AND METHOD}

To obtain information on variations in the GCR planetary system, we have used data from the worldwide network of neutron monitors (41 neutron monitors) [ftp://cr0.irmiran.rssi.ru], corrected for pressure and averaged over hourly intervals, as well as information on electromagnetic conditions in interplanetary space during heliospheric and magnetospheric disturb- ances [https://www.solarmonitor.org; http://cdaw.gsfc. nasa.g/CME_list/UNIVERSAL/2015_06/univ2015_06. html; http://omniweb.gsfc.nasa.gov] with the SGS method [Dvornikov et al., 1986]. Amplitudes of CR modulation were measured from the background level on June 2, 2015.

The statistical error in determining GCR variations from data acquired at the worldwide network of $\mathrm{CR}$ stations with the SGS method is $\sim \pm 0.05 \div 0.07 \mathrm{GV}$.

Basing on the results of calculations of the relationship of GCR variations with GCR derived from data obtained at the worldwide network of CR stations $\Delta R_{\mathrm{ob}}$ and GCR variations with GCR estimated using the axisymmetric model of Earth's limited magnetosphere $\Delta R_{\text {cal }}$ (Kichigin, Sdobnov, 2017), we compute some parameters of magnetospheric current systems (DCF and DR). These parameters in this model are ring current radii $r_{\mathrm{c}}$, magnetopause currents $r_{\mathrm{m}}$, and Dst index. The parameters of current systems $r_{\mathrm{c}}, r_{\mathrm{m}}$ are determined by selecting them to minimize the functional.

$$
\left(\sum_{i}\left[\Delta R_{\mathrm{ob}}\left(R_{i}\right)-\Delta R_{\mathrm{cal}}\left(R_{i}, r_{\mathrm{c}}, r_{\mathrm{m}}, D s t\right)\right]^{2}\right)=\min .
$$

\section{RESULTS}

For the event in June 2015, Figure 1 shows amplitudes of $\mathrm{CR}$ intensity variations and time variations in GCR $\Delta R_{\mathrm{c}}$ in Irkutsk $\left(R_{\mathrm{c}}=3.66 \mathrm{GV}\right)$ (blue curve) together with the Dst index (red curve). The maximum decrease in the intensity of the CR neutron component in Irkutsk in June was $\sim-8 \%$. There is a satisfactory correlation between time variations in $\Delta R_{\mathrm{c}}$ and Dst for June 22-28, 2015; the correlation coefficient is $\sim 0.82$.

Figure $2, a-c$ presents the relationships between GCR variations and GCR for different stages of development of the magnetic storm in June 2015. The variations are calculated from $\mathrm{CR}$ observational data from the worldwide network of stations (blue curve). This Figure also shows the results of calculation of GCR variations using the axisymmetric model of Earth's limited magnetosphere with ring current [Kichigin, Sdobnov, 2017] (red curve). In separate panels are dates and universal time of these relationships, and parameters $\left(r_{\mathrm{c}}-\right.$ the ring current radius, $r_{\mathrm{m}}$ - the magnetosphere radius, and the calculated Dst_cal index) of current systems in the model of Earth's limited magnetosphere with ring current, with which the calculation results obtained by this model are in the closest agreement with those derived from data from the worldwide network of CR stations. There are also observable values of the Dst_ob index.

This Figure indicates that for given parameters of current systems, the GCR variations calculated from CR observations made at the worldwide network of stations and with the model of Earth's limited magnetosphere with ring current, are largely in good agreement, except for some periods of the main phase (see Figure 2,a) and close periods of the magnetic storm under study.

The difference between calculated and observed Dst indices is from several $\mathrm{nT}$ to several tens of $\mathrm{nT}$. The mean radii of the ring current in units of Earth's radius as shown in Figure 2, $a-c$ are $r_{\mathrm{c}}=\sim 3.6$ for the initial phase of the 2015 June geomagnetic storm, $r_{\mathrm{c}}=\sim 4.2$ for the main phase, and $r_{\mathrm{c}}=\sim 4.3$ for the recovery phase. The 

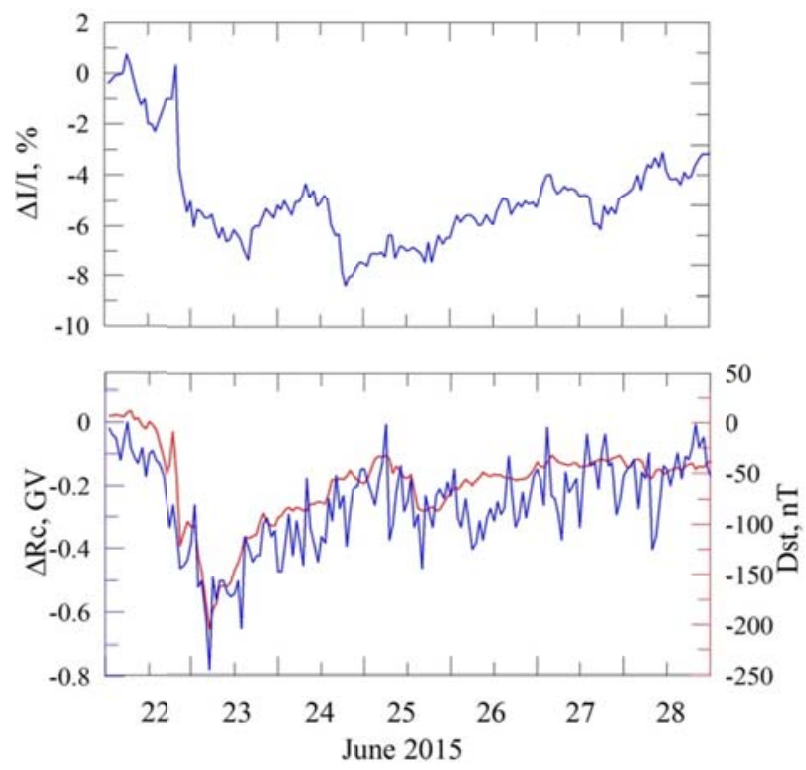

Figure 1. CR intensity amplitude variations $\Delta I / I$ (top); time variations in GCR $\Delta R_{\mathrm{c}}$ in Irkutsk $\left(R_{\mathrm{c}}=3.66 \mathrm{GV}\right)$ (blue curve) together with the Dst index (red curve) for the 2015 June event

mean distance to the subsolar point in the axisymmetric model of the limited magnetosphere for different phases of the 2015 June geomagnetic storm is $r_{\mathrm{m}}=\sim 8.3$ for the initial phase, $r_{\mathrm{m}}=\sim 8.5$ for the main phase, and $r_{\mathrm{m}}=\sim 8.7$ for the recovery phase. The minimum radii of the ring current are observed in the main phase of the geomagnetic storm, followed by an increase in the recovery phase; and the distance to the subsolar point is minimum in the initial phase of the geomagnetic disturbance.

The key feature of a magnetic storm is a sharp increase in the ring current due to injection of charged particles from the magnetotail. During the magnetic storm, along with this increase there is a significant enhancement of other magnetospheric current systems: magnetotail currents, magnetopause currents, and fieldaligned and ionospheric currents which are disregarded in our model. The relationship between contributions of these current systems to the Dst index in different storm phases can characterize their relative dynamics during magnetospheric disturbances.

These radii of the ring current and magnetopause current reflect the total contributions of several current systems. Hence, it is more correct to talk about effective radii of current systems that contribute to the Dst index as the ring and magnetopause currents do.

The estimation of the relative contribution of magnetospheric current systems to the variation of the magnetic field measured on Earth's surface during magnetic storms is currently central. The relationship between Dst sources varies in different phases of magnetic storm development and can describe the relative dynamics of magnetospheric current systems during disturbances.

As for the magnetospheric current systems different from the ring current, there are no standard experimental techniques that allow estimating their contributions to the Dst index. Although measurements made with geostationary instruments provide rough estimates for variation of the current sheet [Ohtani, 2001], they do not allow unambiguous separation of contributions of the main magnetospheric current systems composing the magnetospheric magnetic field. These contributions can be calculated using the currently available magnetospheric models, which allow us to compute separately the field of each magnetospheric source [Alexeev et al., 1996]. Modern models correctly predict the behavior of the magnetic field in both quiet and disturbed periods.
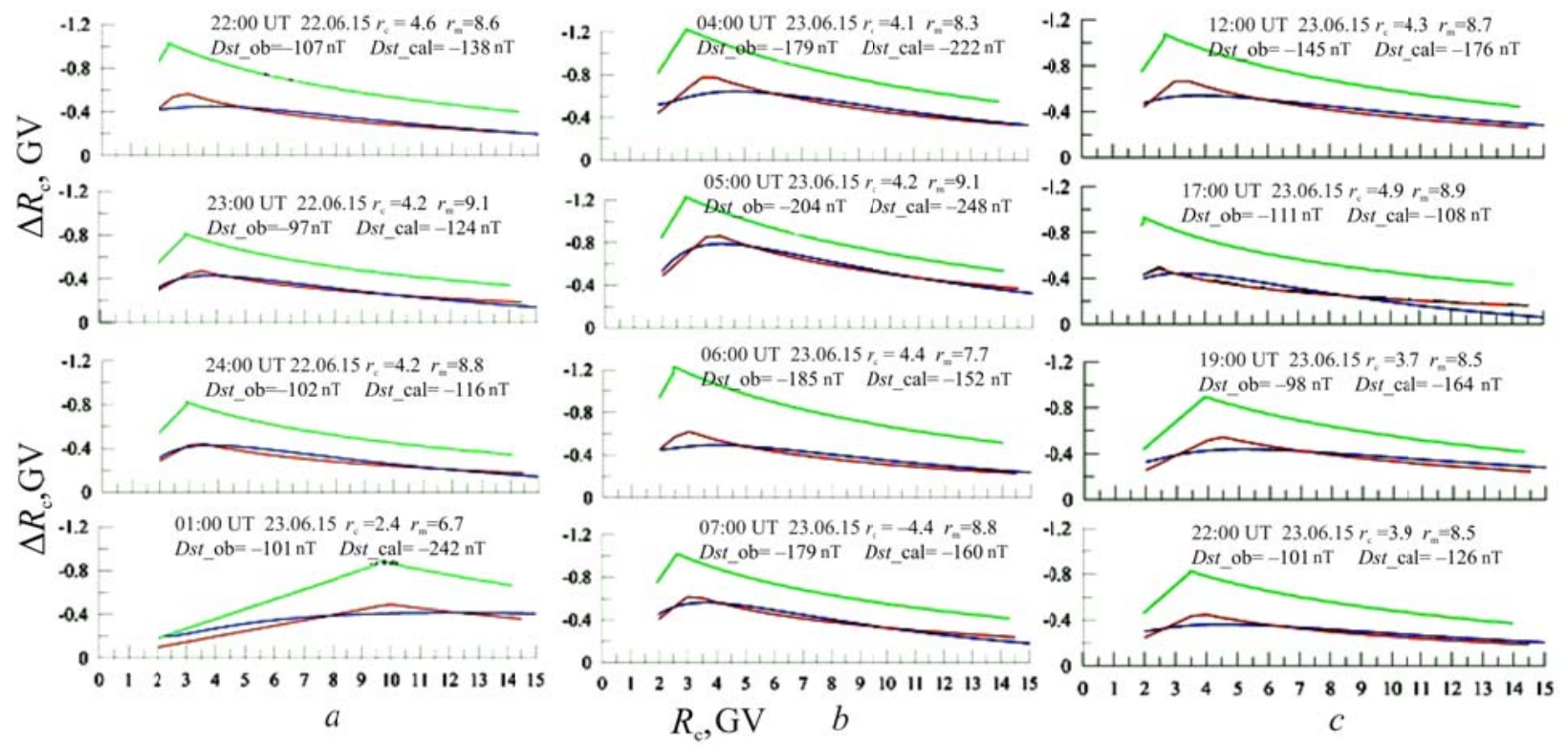

Figure 2. GCR variation vs GGR for different phases of the moderate geomagnetic storm in June 2015: the initial phase (a); the main phase $(b)$; the recovery phase $(c)$. The blue curve shows the results obtained from data acquired at the worldwide network of CR stations with the SGS method; the red curve is the calculation made with the axisymmetric model of the limited magnetosphere with ring current. The green curve is the ring current contribution to GCR variations, estimated using the axisymmetric model of the limited magnetosphere 
At the same time, giving an adequate representation for the Dst-index profile, models often provide very different estimates of the contribution of magnetospheric sources to Dst.

Alexeev et al. [2001] indicate that the differences in estimating the contribution of the magnetotail current sheet to Dst during magnetic storms are often associated with the lack of a unified method for calculating the Dst index based on model calculations. Accordingly, authors determine contributions of magnetospheric current systems to Dst in different ways. Moreover, characteristics of models, their domain of applicability (for empirical models), and the relationships used for parametrization (for theoretical models) can significantly affect relationships between magnetic fields from different sources during a magnetic storm.

Reasons for the discrepancies may also have a physical nature, which is associated with the development of magnetic storms of different intensities under varying conditions in the interplanetary medium and with the prehistory of magnetospheric conditions. The variety of conditions in the interplanetary medium determines the complex dynamics of magnetospheric current systems and hence of the measured magnetic field. In this case, variations in the magnetic field during magnetic storms of different intensity can feature different ratios of contributions of the main magnetospheric current systems.

In this paper, we estimate the contribution of the effective ring current to GCR variations, basing on parameters of current systems in the axisymmetric model of Earth's limited magnetosphere during magnetic storms in June 2015. In Figure 2, this contribution is indicated by a dashed-dot line. The contribution of the ring current to GCR variations is not less than $\sim 50-60$ $\%$ at different storm phases, and the contribution to Dst is at least $60-70 \%$.

\section{CONCLUSIONS}

Ground-based measurements of $\mathrm{CR}$ at the worldwide network of CR stations have been used to estimate GCR variations during geomagnetic disturbances in June 2015. From the GCR variations calculated with the axisymmetric model of Earth's limited magnetosphere, which takes into account the ring current and magnetopause currents, we have determined parameters of current systems, such as the distance to the subsolar point and the ring current radius, as well as the Dst index. Using the axisymmetric model of Earth's limited magnetosphere, we have assessed the contribution of the ring current to GCR variations and to the Dst index.

This study has demonstrated that ground-based CR measurements at the worldwide network of stations can be used to numerically assess parameters of some current systems in the magnetosphere during magnetic storms.

The work was supported by Fundamental Research Program of the RAS Presidium No. 23 "High-Energy Physics and Neutrino Astrophysics" under the project "Cosmic Rays in Heliospheric Processes as inferred from Ground and Stratospheric Observations". Experimental data have been acquired with the Angara Multiaccess Center facilities at ISTP SB RAS and the Unique Scientific Facility (USF) "Russian National Ground-Based Network of Cosmic-Ray Stations" (CRS Network).

We are grateful to S.B. Lunyushkin for the interest in the work, useful discussions and valuable comments in preparation of the manuscript.

\section{REFERENCES}

Alexeev I.I., Belenkaya E.S., Kalegaev V.V., Feldstein Y.I., Grafe A. Magnetic storms and magnetotail currents. J. Geophys. Res. 1996, vol. 101, pp. 7737-7747.

Alexeev I.I., Kalegaev V.V., Belenkaya E.S., Bobrovnikov S.Yu., Feldstein Ya.I., Gromova L.I. The model description of magnetospheric magnetic field in the course of magnetic storm on January 9-12, 1997. J. Geophys. Res. 2001, vol. 106, pp. 25683-25694.

Dvornikov V.M., Sdobnov V.E., Sergeev A.V. Informativity of the Spectrographic Global Survey Method. Preprint SibIZMIR N 25-84. Irkutsk, 1984. 26 p. (In Russian).

Dvornikov V.M., Sdobnov V.E., Sergeev A.V. Spectrographic Global Survey method for research into variations of intensity of cosmic rays of interplanetary and magnetospheric origins. Variatsii kosmicheskikh luchei i issledovaniya kosmosa: Sbornik nauchnykh trudov [Cosmic Ray Variations and Space Research: Collected papers]. Moscow, IZMIRAN Publ., 1986. pp. 232-237. (In Russian).

Feldstein Y.I., Levitin A.E., Kozyra J., Tsurutani B.T., Prigancova A., Alperovich L., Gonzalez W.D., Mall U., Alexeev I.I., Gromova L.I., Dremukhina L.A. Self-consistent modeling of the large-scale distortions in the geomagnetic field during the 24-27 September 1998 major magnetic storm. J. Geophys. Res. 2005, vol. 110, no. A11. DOI: 10.1029/ 2004JA010584.

Ganushkina N.Yu., Pulkkinen T.I., Kubyshkina M.V., Singer H.J., Russell C.T. Long-term evolution of magnetospheric current systems during storms. Ann. Geophys. 2004, vol. 22. pp. 1317-1334.

Greenspan M.E., Hamilton D.C. A test of the DesslerParker-Sckopke relation during magnetic storms. J. Geophys. Res. 2000, vol. 105, pp. 5419-5430.

Kalegaev V.V., Ganushkina N.Yu., Pulkkinen T.I., Kubyshkina M.V., Singer H.J., Russell C.T. Relation between the ring current and the tail current during magnetic storms. Ann. Geophys. 2005, vol. 26, no. 2, pp. 523-533.

Kichigin G.N., Sdobnov V.E. Rigidities of geomagnetic cutoff of cosmic rays in the model of bounded magnetosphere with a ring current. Geomanetizm i aeronomiya [Geomagnetism and Aeronomy]. 2017, vol. 57, no. 2, pp. 149-153. (In Russian).

Maltsev Y.P., Arykov A.A., Belova E.G., Gvozdevsky B.B., Safargaleev V.V. Magnetic flux redistribution in the storm time magnetosphere. J. Geophys. Res. 1996, vol. 101, pp. 7697-7707.

Ohtani S., Nose M., Rostoker G., Singer H., Lui A.T.Y., Nakamura M. Storm-substorm relationship: Contribution of the tail current to Dst. J. Geophys. Res. 2001, vol. 106, pp. 21199-21209.

Tyasto M.I., Danilova O.A., Sdobnov V.E. Comparison of cosmic ray cutoff rigidities as calculated with two empirical magnetospheric models for the extreme event of November 2003. J. Phys .: Conference Ser. 2013, 409, 012224. DOI: 10.1088/17426596/409/1/012224.

Tyasto M.I., Danilova O.A., Sdobnov V.E. Variations in the geomagnetic cutoff rigidity of cosmic rays in the period of magnetospheric disturbances of May 2005: Their correlation with interplanetary parameters. Bull. Russ. Acad. Sci. Phys. 
2011, 75: 808. DOI: 10.3103/S1062873811060414.

URL: ftp://cr0.irmiran.rssi.ru (accessed May 5, 2017).

URL: https://www.solarmonitor.org (accessed May 5, 2017).

URL: http://omniweb.gsfc.nasa.gov (accessed May 5, 2017).

URL: http://cdaw.gsfc.nasa.g/CME_list/UNIVERSAL/2015

_06/univ2015_06.html.

How to cite this article

Kichigin G.N, Kravtsova M.V., Sdobnov V.E. The parameters of the current systems in the magnetosphere according to observations of cosmic rays during the 2015 June magnetic storm. Solar-Terrestrial Physics. 2017. Vol. 3, iss. 3. P. 13-17. 